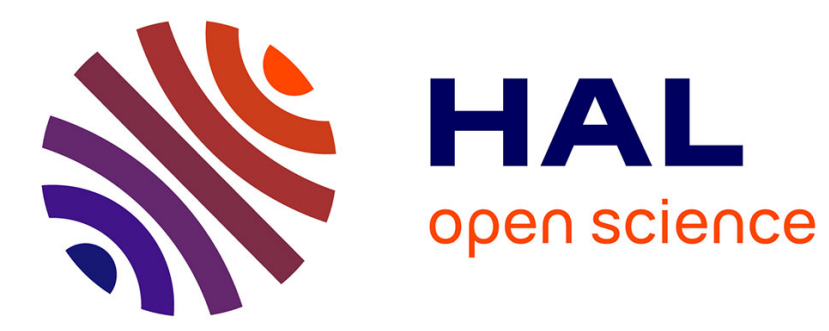

\title{
Relations entre les coefficients thermiques et thermo-élastiques des corps
}

\author{
A. Cornu
}

\section{To cite this version:}

A. Cornu. Relations entre les coefficients thermiques et thermo-élastiques des corps. J. Phys. Theor. Appl., 1873, 2 (1), pp.41-50. 10.1051/jphystap:01873002004100 . jpa-00236905

\section{HAL Id: jpa-00236905 https://hal.science/jpa-00236905}

Submitted on 1 Jan 1873

HAL is a multi-disciplinary open access archive for the deposit and dissemination of scientific research documents, whether they are published or not. The documents may come from teaching and research institutions in France or abroad, or from public or private research centers.
L'archive ouverte pluridisciplinaire HAL, est destinée au dépôt et à la diffusion de documents scientifiques de niveau recherche, publiés ou non, émanant des établissements d'enseignement et de recherche français ou étrangers, des laboratoires publics ou privés. 


\section{RELATIONS ENTRE LES COEFFIGIENTS THERMIQUES ET THERMO-ÉLASTIQUES DES CORPS ;}

PAR M. A. CORNU.

L'étude des phénomènes élastiques et calorifiques que présentent les corps ont conduit les physiciens à la considération de coefficients particuliers, tels que les coefficients d'élasticité, les coefficients de dilatation sous pression constante ou sous volume constant, les caloriques spécifiques, etc., qui tous répondent à certaines circonstances simples de l'observation. Il est résulté de ces recherches particulières une variété de coefficients qui engendre parfois la confusion, car ces coefficients sont loin d'être distincts. Le but de la présente Note est d'établir les relations qui existent entre eux, de rétablir la symétrie de leurs définitions, et finalement d'en réduire le nombre au minimum. Comme on va le voir, ces relations sont de deux natures : les unes sont indépendantes de toute théorie particulière et découlent comme conséquences nécessaires des définitions; les autres s'introduisent comme conséquences des principes de la thermodynamique et peuvent servir de vérification à cette théorie.

Remarque préliminaire. - Tous les coefficients que nous allons étudier sont des limites de rapports : ce sont les quotients de la variation d'une certaine quantité prise comme fonction (volume, quantité de chaleur, etc.) par la variation correspondante d'une autre quantité prise comme variable indépendante (température, pression, etc.). Ce sont donc des dérivées, et même des dérivées partielles, car on suppose que toutes les autres variables du problème restent constantes; il ne faut donc pas oublier que tous ces coefficients définis parfois comme ayant une valeur numérique fixe sont des fonctions de toutes les variables de la question, et ne peuvent être considérés comme constants que dans une trèspetite étendue de l'échelle de variation de ces variables.

\section{DÉFINITIONS DES PRINCIPAUX COEFFICIENTS.}

I. Coefficients déduits de la fonction caractéristique des corps. - On admet que les trois données caractéristiques de l'état d'un II. 
corps quelconque, température $t$, volume $v$ de l'unité de poids, et jression $p$ par unité de surface, sont liées par une relation

$$
\varphi(p, v, t)=0 .
$$

Cette relation générale $\varphi=0$ n'est guère connue que pour les gaz parfaits; elle est égale dans ce cas à

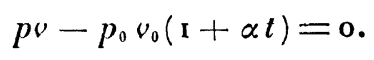

Toutefois on peut, à défaut de la connaissance de cette fonction, se contenter de la différentielle

$$
\frac{\partial \varphi}{\partial p} d p+\frac{\partial \varphi}{\partial v} d v+\frac{\partial \varphi}{\partial t} d t=0
$$

qui lie les variations simultanées $d p, d v, d t$.

On démontre aisément que les cocfficients de dilatation et de compressibilité déterminent les valeurs numériques relatives des trois coefficients différentiels. En effet, on définit le coefficient de dilatation cubique par la formule empirique

$$
v=v_{0}(\mathrm{I}+k t) \text { ou mieux } v=v_{0}[\mathrm{i}+f(t)],
$$

d'où l'on tire

$$
d v=v_{0} \frac{\partial f(t)}{\partial t} d t
$$

Mais il faut ajouter $d p=o$, car la pression est supposée constante; or l'expression $\frac{\mathbf{I}}{v_{0}} \frac{\partial v}{\partial t}$ est le coefficient vrai de dilatation correspondant à la température $t$, et rapporté au volume $v_{0}$ du corps à la température $t=0$. Mais il vaut mieux rapporter la dilatation au volume actuel $v$ correspondant à la température $t$; désignons donc par a le coefficient vrai de dilatation sous pression constante rapporté au volume $v$, non plus au volume $v_{0}$; on aura

$$
d v=v \alpha d t \text { avec } d p=0 .
$$

Substituant ces valeurs relatives de $d p, d v, d t$ dans la relation (2), il vient, en remarquant que le rapport de $d v$ à $d t$ est une dérivée partielle,

$$
v \alpha=\frac{\partial v}{\partial t}=-\left(\frac{\partial v}{\partial t}\right):\left(\frac{\partial v}{\partial v}\right)
$$


De la même manière, le coefficient de compressibilité, d'ordinaire défini par la formule empirique

$$
v=v_{0}(\mathrm{r}-\mu p), \quad \text { ou mieux } v=v_{0}[\mathrm{r}-\psi(p)],
$$

peut être défini d'une manière plus générale par la condition

$$
d v=-v \mu d p \quad \text { avec } \quad d t=0 ;
$$

d'où, par substitution

$$
v \mu=-\frac{\partial v}{\partial p}=\left(\frac{\partial \varphi}{\partial p}\right):\left(\frac{\partial \varphi}{\partial v}\right)
$$

La relation différentiée peut donc être mise sous la forme

$$
\mu d p+\frac{d v}{v}-\alpha d t=0,
$$

relation très-importante qui n'est autre que la différentielle de la fonction $\varphi(p, v, t)=o$, que l'on ne connaît pas en général pour un corps quelconque $\left({ }^{1}\right)$.

On considère aussi quelquefois le coefficient de dilatation sous volume constant déduit de la formule

$$
p=p_{0}(\mathbf{1}+\beta t)
$$

ou

$$
d p=p_{0} \beta d t \text { avec } d v=0 .
$$

Désignant alors par $\beta$ le coefficient vrai de dilatation sous volume constant, on aura

$$
d p=p \beta d t \text { avec } \quad d v=0 .
$$

La substitution dans l'équation (2) conduit à la relation

$$
p \beta=\frac{\partial p}{\partial t}=-\left(\frac{\partial \varphi}{\partial t}\right):\left(\frac{\partial \varphi}{\partial p}\right),
$$

qu'on peut remplacer par la définition du coefficient sous pression

(1) 11 ne faut pas oublier la différence qui existe entre les définitions ci-dessus et les définitions empiriques ordinairement adoptées : ces dernières ont l'inconvénient de supposer inutilement une origine fixe, comme $p_{0}, v_{0}$. Toutefois, au point de vue numérique, la différence, sauf pour les gaz, est souvent négligeable. 
constante, de sorte que l'équation $d \varphi=$ o se met sous la forme

$$
d p+\frac{d v}{v \mu}-p \beta d t=0 .
$$

II. Coefficients calorimétriques. - Lorsqu'on cède une quantité de chaleur infiniment petite $d Q$ à l'unité de poids d'un corps, en général, les trois caractéristiques $p, v, t$ varient infiniment peu; les quatre variations $d \mathrm{Q}, d p, d v, d t$ sont nécessairement liées par une relation linéaire

$$
d \mathbf{Q}=\mathbf{A} d p+\mathbf{B} d v+\mathrm{C} d t
$$

Mais, comme les trois variables $p, v, t$ sont liées par la relation $\varphi=0$, on peut toujours supposer que l'une d'elles a été éliminée, et mettre la valeur de $d Q$ sous l'une des trois formes

$$
\left\{\begin{array}{ccc}
d \mathrm{Q}=c d t+l d v & \text { en éliminant } & p, \\
d \mathrm{Q}=\mathrm{C} d t+h d p & " & v, \\
d \mathrm{Q}=\lambda d v+k d p & \text { " }
\end{array}\right.
$$

\begin{tabular}{|c|c|c|c|c|}
\hline & $\mathrm{C}$ et $h$ & $n$ & $p$ & $n$ \\
\hline ) & $\lambda$ et $k$ & ") & $p$ & ") \\
\hline
\end{tabular}

les coefficients $c$ et $l$ étant fonctions de $v$ et de $t\left({ }^{1}\right)$,

On reconnait dans les coefficients $c$ et $\mathrm{C}$ les capacités calorifiques $\grave{a}$ volume constant et $\grave{a}$ pression constante, c'est-à-dire

$$
\begin{aligned}
& c=\lim \frac{d \mathrm{Q}}{d t} \quad \text { correspondant ̀̀ } \quad d v=\mathbf{o}, \\
& \mathrm{C}=\lim \frac{d \mathrm{Q}}{d t} \quad \text { » } \quad d p=0 .
\end{aligned}
$$

Les coefficients $l$ et $h$ ont été appelés, avant la découverte du principe d'équivalence, chaleurs latentes de dilatation, pour une variation de volume ou de pression. Ce sont, en effet, les rapports

(1) Avant la découverte des principes de la thermodynamique, on aurait ajouté : et de $Q$, c'est-à-dire de la quantité absolue de chaleur que contient le corps. On sait que les trois variables $p, v, t$ suffisent en général pour définir l'état d'un corps quelconque. 
limites des quantités de chaleur $d Q$ dégagées par l'unité de poids du corps, pour une variation de volume $d v$ ou de pression $d p$ sous température constante :

$$
\begin{aligned}
& l=\lim \frac{d Q}{d v} \text { avec } \quad d t=\mathrm{o}, \\
& h=\lim \frac{d Q}{d p} \quad \text { avec } \quad d t=0 .
\end{aligned}
$$

Quant aux coefficients $\lambda$ et $k$, ils n'ont reçu aucun nom; pourtant leur détermination physique directe serait au moins aussi aisée que celle des quatre autres : l'équation (5) les définit suffisamment.

RELATIONS ENTRE LES COEFFICIENTS DÉFINIS CI-DESSUS.

I. Relations indépendantes de toute théorie. - $\mathrm{I}^{\circ}$ L'identification des deux formes (3) et (4) de la relation $d \varphi=0$ exige que l'on ait

$$
\alpha=\beta \mu p,
$$

équation qui établit une relation très-simple entre les deux coefficients de dilatation et le coefficient de compressibilité. $2^{0}$ Les six coefficients calorimétriques $c, \mathrm{C}, l, h, \lambda, k$ ont entre eux quatre relations très-simples, qui proviennent de ce que les trois expressions de $d Q$ doivent se réduire à des identités, en ayant égard à la relation $d \varphi=0$.

En effet, égalant les deux premières expressions de $d \mathbf{Q}$, on a

$$
c d t+l d v=\mathrm{C} d t+h d p ;
$$

les valeurs relatives des trois différentielles étant arbitraires, cette expression, mise sous la forme

$$
h d p-l d v+-(\mathrm{C}-c) d t=0,
$$

ne peut être exacte que si elle est identique avec l'équation $d \varphi=0$,

$$
\mu d p+\frac{d v}{v}-\alpha d t=\mathbf{o},
$$

où les rapports des trois différentielles sont également arbitraires. 
A. CORNU.

L'identification donne deux des relations cherchées

$$
\frac{h}{\mu}=-l v=-\frac{\mathrm{C}-c}{\alpha} .
$$

On obtiendra les deux autres en partant de la deuxième et de la troisième valeur de $d Q$

ou

$$
\mathrm{C} d t+h d p=\lambda d v+k d p,
$$

$$
(h-h) d p+\lambda d v-\mathrm{C} d t=\mathrm{o} .
$$

On en conclut

$$
\frac{k-h}{\mu}=\lambda v=\frac{\mathrm{C}}{\alpha}
$$

Ces quatre relations très-importantes permettent de réduire les six coefficients $c, \mathrm{C}, l, h, \lambda, k$ à deux distincts. Ceux qui offrent l'interprétation la plus simple sont $c$ et $\mathrm{C}$; on va donc exprimer $l$, $h, \lambda, k$ en fonction de $\mathrm{C}, c, \alpha$ et $\mu$, ou encore, par raison de symétrie en fonction de $\alpha \beta$ [en ayant égard à la relation (I) $\alpha=\beta \mu p$ ],

$$
\begin{cases}l=\frac{\mathrm{C}-c}{\alpha v}, & \lambda=\frac{\mathrm{C}}{\alpha v}, \\ h=-\frac{\mathrm{C}-c}{\alpha} \mu=-\frac{\mathrm{C}-c}{\beta p}, & k=h+\frac{\mathrm{C} \mu}{\alpha}=\frac{c}{\beta p} .\end{cases}
$$

Il est bien entendu qu'il faut joindre à ces expressions l'équation $\varphi=0$, ou sa différentielle $d \varphi=0$.

Remarque. - Ces relations sont indépendantes de toute théorie; elles résultent uniquement des définitions et de l'existence de la fonction $\varphi(p, \nu, t)=0$.

II. Relations déduites du principe de l'équivalence de la chaleur et du travail. - Équations de Clausius. - L'expression analytique du principe d'équivalence de la chaleur et du travail permet de nouvelles identifications, qui fournissent trois nouvelles relations entre les coefficients calorimétriques.

Si l'on désigne par $\mathrm{U}$ la fonction de $p, v, t$ qui représente l'énergie interne du corps, par $\tau=\int p d v$ le travail extérieur, et par $\mathrm{E}$ 
l'équivalent mécanique de la chaleur, on a

$$
\mathbf{E} d \mathbf{Q}=d \mathbf{U}+p d v .
$$

Or U peut être exprimé de trois manières ( $\left.{ }^{1}\right)$, suivant qu'on élimine $p, v, t$ à l'aide de l'équation $\varphi=0$. Donc on aura

$$
\begin{aligned}
\mathbf{E} d \mathbf{Q} & =\frac{\partial \mathrm{U}(t, v)}{\partial t} d t+\frac{\partial \mathrm{U}(t, v)}{\partial v} d v+p d v=\mathbf{E}(c d t+l d v) \\
& =\frac{\partial \mathrm{U}(t, n)}{\partial t} d t+\frac{\partial \mathrm{U}(t, p)}{\partial p} d p+p\left(\frac{\partial v}{\partial t} d t+\frac{\partial v}{\partial p} d p\right)=\mathbf{E}(\mathrm{C} d t+h d p) \\
& =\frac{\partial \mathrm{U}(p . v)}{\sigma p} d p+\frac{\partial \mathrm{U}(p, v)}{\partial v} d v+p d v=\mathbf{E}(\lambda d v+k d p) .
\end{aligned}
$$

D'où l'on conclut identiquement

$$
\begin{array}{ll}
\mathbf{E} c=\frac{\partial \mathbf{U}(t, v)}{\partial t}, & \mathbf{E} l=\frac{\partial \mathrm{U}(t, v)}{\partial v}+p, \\
\mathbf{E C}=\frac{\partial \mathbf{U}(t, p)}{\partial t}+p \frac{\partial v}{\partial t}, & \mathbf{E} h=\frac{\partial \mathbf{U}(t, p)}{\partial p}+p \frac{\partial v}{\partial p}, \\
\mathbf{E} k=\frac{\partial \mathbf{U}(p, v)}{\partial p}, & \mathbf{E} \lambda=\frac{\partial \mathbf{U}(p, v)}{d v}+p .
\end{array}
$$

On élimine la fonction $\mathrm{U}$ en remarquant que la dérivée seconde d'une fonction, prise par rapport à deux variables, possède identiquement la même valeur, quel que soit l'ordre des différentiations. Par exemple, dans le cas de $\mathbf{U}(t, v)$, on a deux expressions de $\frac{\partial^{2} \mathbf{U}}{\partial t \partial v}$,

$$
\mathbf{E} \frac{\partial c}{\partial v}=\frac{\partial^{2} \mathbf{U}(t, v)}{\partial t \partial v} \quad \text { et } \quad \mathbf{E} \frac{\partial l}{\partial t}=\frac{\partial^{2} \mathbf{U}(t, v)}{\partial v \partial t}+\frac{\partial p}{\partial t} .
$$

Égalant ces valeurs, et opérant de même avec les deux autres systèmes, on arrive aux trois relations

$$
\left\{\begin{array}{l}
\mathbf{E}\left(\frac{\partial l}{\partial t}-\frac{\partial c}{\partial v}\right)=\frac{\partial p}{d t}=\alpha v \\
\mathbf{E}\left(\frac{\partial \mathrm{C}}{\partial p}-\frac{\partial h}{\partial t}\right)=\frac{\partial v}{\partial t}=p \beta \\
\mathbf{E}\left(\frac{\partial \lambda}{\partial p}-\frac{\partial k}{\partial v}\right)=\mathbf{I}
\end{array}\right.
$$

(1) En écrivant $\mathbf{U}(t, v), \mathbf{U}(t, p), \mathbf{U}(p, t)$, on indique le résultat qu'on obtiendrait après élimination de l'une des trois variables. 
Ces trois nouvelles relations ne sont pas immédiatement utilisables à simplifier le nombre des coefficients thermiques, mais leur importance va apparaître dans ce qui suit.

III. Relations déduites du principe de Carnot. - Équations de W. Thomson. - Le principe de Carnot conduit à écrire que, pour un cycle fermé quelconque, l'intégrale $\int \frac{d Q}{T}$ est nulle ( $T$ étant la température absolue $\left.=t+27^{\circ}\right)$. Cette condition exige que l'expression $\frac{d Q}{T}$ soit une différenticlle exacte des deux variables indépendantes qu'on a choisies.

On sait, en effet, qu'une expression de la forme $\mathrm{X} d x+\mathrm{Y} d y$, $\mathrm{X}$ et $\mathrm{Y}$ étant deux fonctions de $x$ et $y$, n'est pas en général la différentielle totale d'une fonction $\mathbf{F}(x, y)$, mais qu'elle peut le devenir par la multiplication, par une fonction de $x, y, z$, qu'on nomme facteur d'intégrabilité.

L'expression $\mathrm{E} d \mathrm{Q}=d \mathbf{U}+p d v$ n'est pas une différentielle exacte, puisqu'elle se compose de $d U$ qui en est une, et de $p d v$ qui n'en est pas une. Le facteur d'intégrabilité est donc ici $\frac{\mathbf{I}}{\mathbf{T}}$, c'està-dire la valeur tirée de l'équation $\varphi(p, v, t)=0$ résolue par rapport à $\frac{\mathbf{I}}{273^{\circ}+t}$.

On pourra donc exprimer $\frac{d Q}{T}$ de trois manières, d'après les trois valeurs de $d \mathrm{Q}$, en considérant $t$ et $v, t$ et $p$, ou $p$ et $v$, comme variables indépendantes. La condition analytique pour que $\mathrm{X} d x+\mathrm{Y} d y$ soit la différentielle exacte d'une fonction $\mathrm{F}$ est

$$
\frac{\partial \mathbf{X}}{\partial y}=\frac{\partial \mathbf{Y}}{\partial x}=\frac{\partial^{2} \mathbf{F}}{\partial x \partial y}
$$

ce qui revient, comme dans le cas précédent, à identifier les deux expressions d'une dérivée seconde prise par rapport à deux variables

$$
\begin{aligned}
& \frac{d \mathbf{Q}}{\mathbf{T}}=\frac{c}{\mathbf{T}} d t+\frac{l}{\mathbf{T}} d v, \quad \text { d'où } \frac{\partial}{\partial v}\left(\frac{c}{\mathbf{T}}\right)=\frac{\partial}{\partial t}\left(\frac{l}{\mathbf{T}}\right), \\
& =\frac{\mathrm{C}}{\mathrm{T}} d t+\frac{h}{\mathrm{~T}} d p, \quad \frac{\partial}{\partial p}\left(\frac{\mathrm{C}}{\mathrm{T}}\right)=\frac{\partial}{\partial t}\left(\frac{h}{\mathrm{~T}}\right), \\
& =\frac{k}{\mathrm{~T}} d p+\frac{\lambda}{\mathrm{T}} d v, \quad \frac{\partial}{\partial v}\left(\frac{l}{\mathrm{~T}}\right)=\frac{\partial}{\partial p}\left(\frac{\lambda}{\mathrm{T}}\right) ;
\end{aligned}
$$


toutes réductions faites, on trouve

$$
\begin{aligned}
& \mathbf{T}\left(\frac{\partial l}{\partial t}-\frac{\partial c}{\partial v}\right)=l \\
& \mathbf{T}\left(\frac{\partial h}{\partial t}-\frac{\partial c}{\partial v}\right)=h, \\
& \mathbf{T}\left(\frac{\partial h}{\partial v}-\frac{\partial \lambda}{\partial p}\right)=k \frac{\partial t}{\partial v}-\lambda \frac{\partial t}{\partial p} .
\end{aligned}
$$

Ces équations se simplifient beaucoup, si l'on a égard aux équations (V) déduites du premier principe; elles deviennent

$$
\left\{\begin{array}{l}
l=-\frac{\mathbf{I}}{\mathbf{E}} \mathbf{T} \frac{\partial p}{\partial t} \\
\iota=-\frac{\mathbf{I}}{\mathbf{E}} \mathbf{T} \frac{\partial v}{\partial t} \\
k \frac{\partial t}{\partial v}-\lambda \frac{\partial t}{\partial p}=-\frac{\mathbf{I}}{\mathbf{E}} \mathbf{T} .
\end{array}\right.
$$

Substituant à la place des dérivées partielles les valeurs de $\alpha$, $\beta$ et $\mu$, posant en outre $A=\frac{1}{\mathbf{E}}$, il vient

$$
\left\{\begin{array}{l}
l=\mathbf{A T} p \beta, \\
h=-\mathbf{A T} v \alpha, \\
\frac{l}{\alpha v}-\frac{\lambda}{p \beta}=-\mathbf{A T} .
\end{array}\right.
$$

Ces trois nouvelles relations sembleraient devoir réduire de trois unités le nombre des coefficients distincts; il est facile de vérifier qu'elles ne sont pas elles-mêmes distinctes, mais se réduisent à une seule. Éliminant, en effet, AT, il vient

$$
\mathrm{AT}=\frac{l}{p \beta}=-\frac{h}{v \alpha}=-\frac{k}{\alpha v}+\frac{\lambda}{p \beta} .
$$

Substituant les valeurs de $l, h, k, \lambda(\mathbf{I V})$, on a

$$
\mathrm{AT}=\frac{\mathrm{C}-c}{\alpha v p \beta}=\frac{\mathrm{C}-c}{\beta p \alpha v}=-\frac{c}{\alpha v \beta p}+\frac{\mathrm{C}}{\alpha v \beta p} ;
$$


il reste donc la relation unique

$$
\mathrm{C}-c=\mathrm{AT} \alpha \beta \vee p,
$$

s'appliquant à un corps quelconque.

Cette relation nouvelle suppose l'exactitude des deux principes de la Thermodynamique, lesquels paraissent maintenant complétement établis.

En résumé, si l'on veut utiliser toutes les relations démontrées ci-dessus, on peut exprimer tous les coefficients thermiques ou thermo-élastiques en fonction de trois d'entre eux : par exemple, le coefficient de dilatation sous pression constante, le coefficient de compressibilité cubique et la capacité calorifique sous pression constante.

Voici le résultat de l'élimination de $c$, qui n'est pas directement accessible à l'expérience,

$$
\begin{array}{rlrl}
\beta & =\frac{\alpha}{\mu \cdot p}, & c & =\mathrm{C}-\mathrm{AT} \frac{\alpha^{2} v}{\mu} ; \\
l & =\mathbf{A T} \frac{\alpha}{\mu}, & \lambda=\frac{\mathrm{C}}{\alpha v} ; \\
h & =-\mathbf{A T} \alpha v, & k=\frac{\mathrm{C} \mu}{\alpha}-\mathbf{A T} \alpha v .
\end{array}
$$

Ainsi le nombre des coefficients strictement nécessaires est réduit à trois dans le cas simple où le corps est supposé homogène et isotrope, et où la pression extérieure est répartie uniformément sur sa surface. Ce qui revient à dire qu'il reste isotrope dans toutes les modifications qu'il subit. 